\begin{tabular}{|c|c|c|c|c|c|c|c|}
\hline IEEE.org & IEEE Xplore & IEEE-SA & IEEE Spectrum & More Sites & SUBSCRIBE & SUBSCRIBECart Create Account & Personal Sign In $\rightarrow$ \\
\hline & & & Browse V & My Settings $V$ & Help V & Institutional Sign In & \\
\hline
\end{tabular}

All

\title{
On the Secrecy Performance of NOMA-Based Integrated Satellite Multiple-Terrestrial Relay Networks With Hardware Impairments
}
Publisher: IEEE
Cite This
$\Delta$ PDF
Kefeng Guo
; Kang An
; Feng Zhou
; Theodoros A. Tsiftsis
; Gan Zheng
; Symeo..
All Authors
3
Paper $\quad 347$
Citations $\quad$ Text Views

\section{Abstract}

Document Sections

I. Introduction
Abstract:Integrated satellite terrestrial networks and non-orthogonal multiple access (NOMA) have been confirmed to be promising and effective approaches to achieve substantial pe... View more

\section{- Metadata}

Abstract:

\section{More Like This}

NOMA-based Integrated SatelliteTerrestrial Multi-relay Networks with Hardware Impairments and Partial Relay Selection Scheme 2019 IEEE 19th International Conference on Communication Technology (ICCT)

Published: 2019 
II. System Model and Problem

Formulation

III. Performance Analysis

IV. Secrecy Energy Efficiency

V. Numerical Results

Show Full Outline Authors

Figures

References

Citations

Keywords

Metrics

More Like This

Footnotes
Integrated satellite terrestrial networks and non-orthogonal multiple access (NOMA) have been confirmed to be promising and effective approaches to achieve substantial performance gains for future wireless paradigms. This paper studies the effect of hardware impairments (HIs) on the secrecy performance of NOMA-based integrated satellite multiple-terrestrial relay networks (ISMTRNs). Particularly, we consider two important wiretapping cases: Case I, colluding case: all eavesdroppers cooperatively overhear the information; and Case II, non-colluding case: one eavesdropper with the best wiretapping quality is chosen to overhear the main channel information. Specially, the closed-form expressions for the secrecy outage probability (SOP) of the considered NOMA-based ISMTRNs in the presence of the above two eavesdropping cases and partial relay selection scheme are obtained. To obtain further insights in high signal-tonoise ratios (SNRs) regime, the asymptotic analysis of SOP with two considered cases are also derived, which give efficient means to evaluate the benefit of NOMA scheme and the impacts of HIs on the SOP. Moreover, we obtain Monte Carlo (MC) simulations for the secrecy energy efficiency (SEE).

Published in: IEEE Transactions on Vehicular Technology ( Volume: 70 , Issue: 4, April 2021)

Page(s): $3661-3676$

DOI: 10.1109/TVT.2021.3068062

Date of Publication: 23 March 2021 ? Publisher: IEEE

\section{- ISSN Information:}

D Funding Agency:
Secrecy Performance Analysis of NOMA-Based Integrated SatelliteTerrestrial Relay Networks with Multiple Colluding Eavesdroppers 2020 International Conference on Wireless Communications and Signal Processing (WCSP)

Published: 2020

\section{Show More}

\section{:三 Contents}

I. Introduction

The integration of satellite Sign in to Continue Reading 
terrestrial mobile networks is one of the key element for the sixthgeneration (6 G) and next generation networks due to enhanced capability and widely covered area [1], [2]. SatCom has been utilized in many fields, such as rescue, broadcasting and broadband because it can bring the high reliability, reduce the latency, enlarge the coverage area and give low-cost and energy-efficient methods [3]. However, the coverage region may be reduced by the obstacles and shadowing between the terrestrial users and satellite, which results in the unavailability of the line-of-sight (LOS) links [4]. On this condition, the terrestrial relay is utilized to solve this shortage [5], which leads to the framework of the integrated satellite-terrestrial relay networks (ISTRNs). The main idea of ISTRNs is to utilize terrestrial relays to forward the signal of the satellite, which is becoming a promising architecture for the SatCom, especially for the fixed satellite service and mobile satellite service, which has been used in the real systems such as SiriusXM [6]. In fact, ISTRN has been incorporated in Digital Video Broadcasting (DVB) system which provides Satellite services to Handheld devices $(\mathrm{SH})$, leading to the standard known as DVB-SH [7], ISTRN has been concluded in "Space-Ground Integrated Information Network Engineering" of China [8].

\begin{tabular}{lr}
\hline Authors & $\checkmark$ \\
\hline Figures & $\checkmark$ \\
\hline References & $\checkmark$ \\
\hline Citations & $\checkmark$ \\
\hline Keywords & $\checkmark$ \\
\hline Metrics & $\checkmark$ \\
\hline Footnotes & $\checkmark$ \\
\hline
\end{tabular}




\section{IEEE Personal Account}

CHANGE USERNAME/PASSWORD

\section{Purchase Details}

PAYMENT OPTIONS

VIEW PURCHASED DOCUMENTS
Profile Information

COMMUNICATIONS PREFERENCES

PROFESSION AND EDUCATION

TECHNICAL INTERESTS

\section{Need Help?}

US \& CANADA: +1 8006784333

WORLDWIDE: +1 7329810060

CONTACT \& SUPPORT

About IEEE Xplore | Contact Us | Help | Accessibility | Terms of Use | Nondiscrimination Policy | IEEE Ethics Reporting $[\boldsymbol{~}$ | Sitemap | Privacy \& Opting Out of Cookies A not-for-profit organization, IEEE is the world's largest technical professional organization dedicated to advancing technology for the benefit of humanity.

(c) Copyright 2021 IEEE - All rights reserved. Use of this web site signifies your agreement to the terms and conditions.

\section{IEEE Account}

»Change Username/Password

» Update Address

\section{Purchase Details}

»Payment Options

»Order History

»View Purchased Documents

\section{Profile Information}

»Communications Preferences

»Profession and Education

» Technical Interests

\section{Follow}

About IEEE Xplore | Contact Us | Help | Accessibility | Terms of Use | Nondiscrimination Policy | Sitemap | Privacy \& Opting Out of Cookies

A not-for-profit organization, IEEE is the world's largest technical professional organization dedicated to advancing technology for the benefit of humanity. (c) Copvriaht 2021 IEEE - All riahts reserved. Use of this web site sianifies vour aqreement to the terms and conditions. 